\title{
NEW APPROACH FOR NOISE CLEANING ON REAL TIME VIDEOS BASED LOW-PASS FILTERING AND MATHIMATICAL MORPHOLOGY
}

\author{
Dhekra ESSAIDANI, Hassene SEDDIK and Ezzedine BEN BRAIEK \\ dhekraessaidani89@gmail.com, hassene.seddik@estt.rnu.tn and ebenbraiek@yahoo.fr
}

\begin{abstract}
Video de-noising presents a necessary step in video processing. It improves its quality and permits a preview processing for other applications such as objects tracking, compression, feature extraction, edge detection, motion tracking...etc.

In this paper, we combined the mathematical morphology operations with low-pass filtering to improve real-time video de-noising corrupted by different noise types. Different kinds of morphologies and filters are proposed in order to define the best combination possible. This algorithm does not require any motion estimation. It isn't present a blur in de-noising video. Nevertheless, it can be a good reference for estimate motion in video.
\end{abstract}

\section{Key words}

Real time video de-noising, low pass filter, mathematical morphology, video filtering, motion estimation.

\section{INTRODUCTION}

Image quality enhancement is a long-standing area of research. Image and video capture devices, such as web-cams and cell phones, become omnipresent. This creates a need for efficient digital image and video enhancement technologies to improve their outputs. Noise is a main factor that degrades image quality. It reduces in video sequences compression performance. Many algorithms are proposed in the literature for noise removal applying a spatiol-temporal filtering [6]. M. Ghoniem, has integrated temporal and spatial coordinate of the successive images to develop a spatiotemporal regularization on weighted graphs [6]. A. Acharya combined high boost filter with soft filter for video de-noising with preserving fines detail of the processed objects [1]. Many other techniques combine wavelet spatial de-noising and temporal filtering. E. J. Balster, proposed to combine theses two domains for wavelet shrinkage applied on noisy video [8]. Other techniques used motion estimation to establish temporal correspondence across frames in order to achieve high-quality video de-noising [1, 6, 15, 8 and 10].

V. Gijesh combined space-time vector of the wavelet coefficients and spatiotemporal Gaussian scale mixture to replicate the effect of motion [11]. Sezan proposed motion compensation based spatio-temporal linear minimum mean square error filter (LMMSE [14]. Nearly, recent works have shown that video denosing by resolving the motion estimation may induce damaging effects [2]. On the other hand digital video pre-processing aims to eliminate the image noise that reduces the overall compression performance while preserving the visual quality of the image sequence. An efficient kind of pre-processing is mathematical morphologies [10]. Mathematical morphology represents a nonlinear approach applied as pre-processing tool for signal processing [10]. It is based on the application of lattice theory to spatial structures. This technique is proven to be a very powerful tool in image analysis [4].
Combining mathematical morphology with low-pass filters for video de-noising, the proposed approach does not require any motion estimation to de-noise videos. Using the morphology as peprocessing tool, it offers a prior prepare of the images frames processed by different de-noising filters. The simulation results proved the high improvements when the morphological processing is applied. The video is well de-noised face to several noise kinds. This method stays adequate for motion estimation.

This paper is organized as follows: in section 2 the mathematical background is developed. Experimental results are validated in section 3, Discussions and experimental results are provided in section 4 and then we conclude the paper.

\section{PROPOSED METHOD}

In video de-noising, motion remains the fundamental problem. Standard low pass filter is not able in this treatment. It can lose details and produces blur which introduces high dissimilarity between frames. In the literatures compensated filters are proposed combined with motion estimation or combined filters such as Winner and Kalman filter. This proposed approach is based on combining mathematical morphology and low-pass filter for noise reduction. Morphology is widely used as image processing tool and a specific low-pass filter is selected to filter images. But how the combination can be done? Which filter we choose and what is the best morphology and size of its structure element matches the best this filter?

In this approach a data base of 25 real video with $10 \mathrm{MP}$ resolution are used. Three kinds of noise are applied respectively: periodic, Gaussian and salt and pepper noises.

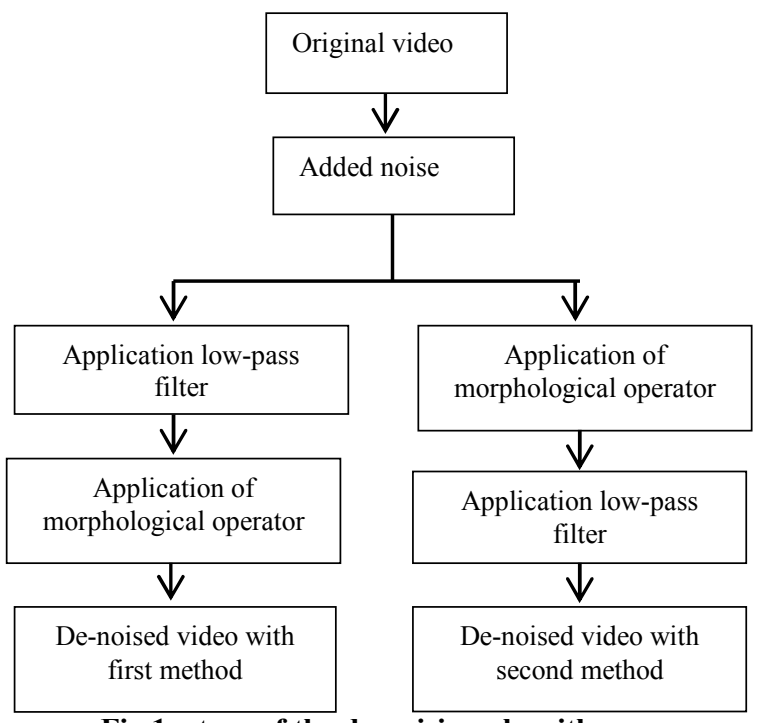

Fig 1: steps of the de-noising algorithm

Where: $\mathrm{MM}$ is the Mathematical morphology, $\mathrm{V}_{\mathrm{o}}$ the original video, $V_{N}$ the Noisy Video and $V_{d I}$ and $V_{d 2}$ the de-noised videos. I the following we will note by $n_{f}$ the total numbers of frames composing the video, $N(x, y, k)$ is the applied noise, $I_{N}$ is the noised image, $\mathrm{I}_{\mathrm{O}}$ is the original image, $I_{D}$ is the de-noised image where $(x, y)$ is the spatial coordinate and $\mathrm{k}$ is the number of frames, $F_{p l}$ is low pass filter 
of size $[L, Q]$ ( $L$ and $Q$ are odd) and the structural element noted by $E(m, n)$.

The second algorithm is the same as the only first, we applied mathematical morphology on noised video and low pass filter on analyzed video by morphological operators.

\section{A. Noises notion}

A real-time video is the result of cascade electronic systems. Technology proves that any electronic component is imperfect. It produces faulty effects on their outputs. These effects conscript noise [12]. In order to study this adverse effect, we modeled our noise by the equation [9]:

$V_{n}(x, y, k)=V_{o}(x, y, k)+N(x, y, k)$

$V_{n}(x, y, k)$ and $V_{o}(x, y, k)$ show respectively the noisy and the original video. $N(x, y, k)$ represent the interference signal. The index $(x, y)$ shows the spatial coordinates of video image while $k$ present the sampling instant of current frame.

\section{B. Low pass video filtering}

In this step, we used low pass filter to reduce noise of real time video. Based on the 2D general expression of low-pass filtering given by the following equation:

$$
I_{D}(x, y)=\frac{\sum_{i=1}^{N} \sum_{j=1}^{M} I_{N}(\lambda-x, \gamma-y) \bullet F_{p l}(x, y)}{\sum_{i=1}^{N} \sum_{j=1}^{M} F_{p l}(x, y)}
$$

We define the equation of low-pass filtering video below:

$$
\begin{aligned}
& V_{f}(x, y, k)=n_{f} \diamond E_{f} \\
& \text { with: } E_{f}=\frac{1}{A}\left[\sum_{i=1}^{N} \sum_{j=1}^{M} V_{N}(\alpha-x, \beta-y, k) \bullet F_{p l}(x, y, k)\right] \\
& \qquad A=\sum_{i=1}^{N} \sum_{j=1}^{M} F_{p l}(x, y, k)
\end{aligned}
$$

The instruction $E_{f}$ is repeated $n_{f}$ times:

$$
n_{f} \diamond E_{f}=\underbrace{\left\{E_{f}, E_{f}, E_{f}, \ldots \ldots \ldots \ldots . . . E_{f}\right\}}_{n_{f} \text { times }}
$$

Where $n_{f}$ represent the total number of frames in the video sequence.

\section{Mathematical morphology}

It is a theory of non-linear image processing based on settheory, structural elements propriety, mathematical operations such as inclusion, intersection, meeting and logical operations to analyze images. This technique analyzes the geometrical characteristic of video objects and video image areas [14].

\section{1) Structural element}

It is a geometry shape characterized by its size, shape, connectivity and origin. It is not necessarily symmetrical. During treatment, its origin must be placed on the processed pixel. It defines the conservation and eliminates areas in processing image [7]. The mathematical morphology filter is defined by the dilation, erosion, opening and closing operation.

\section{2) Dilation}

Dilation is an extensive, increase and not-idempotent transformation. It removes the black points isolated. It fills the valleys and peaks thicken [14]. The expansion of a video frame $I_{v}$ by the structuring element $[B, A, k, j]$ is defined by this equation [10]:

$$
\begin{aligned}
I_{v} \oplus[B, A, k, j](v)=k^{t h}\left\{k \diamond I_{v}(a): a \in A_{v}\right\} \\
\\
\bigcup\left\{j \diamond I_{v}(b): b \in(B-A)_{v}\right\}
\end{aligned}
$$

Based on this equation and the dilation equation of gray level image $I(x, y)$ with the structural element $E(m, r)$ noted $I \oplus E$ and defined in [6] by:

$$
\begin{aligned}
I_{D}(x, y) & =(I \oplus E)(x, y) \\
& =\max \{I(x-m, y-r)+E(m, r)\}
\end{aligned}
$$

For $(m, r) \in E$ and $(x-m, y-r) \in E$.

We define the morphological dilation applied to real time video sequence by this equation:

$$
\begin{aligned}
V_{D D}(x, y, n) & =\left(V_{f} \oplus E\right)(x, y, n) \\
& =\left\{n_{f} \diamond \max ^{n}\left\{V_{f}(x-m, y-r, n)+E(m, r)\right\}\right\}
\end{aligned}
$$

For $(m, n) \in E$ and $(x-m, y-r) \in E$ and $1 \leq n \leq n_{f}$

\section{3) Erosion}

The erosion is anti-extensive, increase and non-idempotent transformation [14]. The erosion of a video frame $I_{v}$ by the structuring element $[B, A, k, j]$ is defined by this equation [10]:

$$
\begin{array}{r}
I_{v} \Theta[B, A, k, j](v)=k^{\text {th }}\left\{k \diamond I_{v}(a): a \in A_{v}\right\} \\
\bigcup\left\{j \diamond I_{v}(b): b \in(B-A)_{v}\right\}
\end{array}
$$

With $k^{\text {th }}$ is the minimum value of the whole. Based on this equation and the equation applied to still image defined in [2] by:

$$
\begin{aligned}
\operatorname{Ie}(x, y)= & (I \Theta E)(x, y) \\
& =\min \{I(x+m, y+r)-E(m, r)\}
\end{aligned}
$$

For $(m, r) \in E$ and $(x-m, y-r) \in E$.

We define the morphological erosion applied to real time video sequence by this equation:

$$
\begin{aligned}
V_{D E}(x, y, n) & =\left(V_{f} \oplus E\right)(x, y, n) \\
& =\left\{n_{f} \diamond \min ^{n}\left\{V_{f}(x+m, y+r, n)+E(m, r)\right\}\right\}
\end{aligned}
$$

For $(m, n) \in E$ and $(x-m, y-r) \in E$ and $1 \leq n \leq n_{f}$

\section{4) Opening}

It is a dilation transformation of an eroded image. It is idempotent, extensive and increase transformation [10]. The open transformation of a video frame $I_{v}$ by the structural element $[B, A, k, j]$ is defined in [15] by this equation:

$$
I_{v_{[B, A, k, j]}}(v)=\left(I_{v} \Theta[B, A, k, j]\right) \oplus\left[B^{s}, A^{s}, k, j\right](v)
$$

Based on this equation, we expressed our open operators by:

$\left.V_{D O}(x, y, n)=\left\{n \diamond \mid\left(V_{f}(x, y, n) \oplus E\right) \Theta E\right]\right\}$

\section{5) Closing}

Closing is an erosion transformation of a dilated image. It is increase, idempotent and extensive transformation [10]. 
Closing of a video frame $I_{v}$ by the structural element $[B, A, k$, $j]$ defined by this equation [15]:

$$
I_{v}^{[B, A, k, j]}(v)=\left(I_{v} \oplus[B, A, k, j]\right) \Theta\left[B^{s}, A^{s}, k, j\right](v)
$$

Based on this equation, we defined the closing operation by this equation:

$$
\left.V_{D C}(x, y, k)=\left\{n_{f} \diamond \mid\left(V_{f}(x, y, k) \Theta E\right) \oplus E\right]\right\}
$$

\section{Measuring quality of filtered video}

We used the Peak Signal to Noise Ratio (PSNR) in decibels value to compare the quality of video images [7 and 3]. It computes Peak Signal to Noise Ratio between two images. The higher PSNR shows better quality of the processed video. The PSNR value computes by this equation [3]:

$$
P S N R=10 \log _{10}\left(\frac{B^{2}}{M S E}\right)
$$

$B$ is the maximum intensity in the input video image. $M S E$ shows the mean square error between the filtered and the noisy image. MSE Value computes in [7] by this equation:

$$
M S E=\frac{\sum_{l=1}^{L} \sum_{c=1}^{C}\left[V_{o}(x, y)-V_{D}(x, y)\right]^{2}}{L^{*} C}
$$

$L$ shows the number of rows and $C$ shows the number of columns of the frame. $V_{D}(x, y)$ represent the denoised frame and $V_{D}(x, y)$ represent the original frame.

\section{THE NEW APPROACH APPLICATION}

In order to define the robustness of our approach, we used three noise types with different intensity. The first is a random noise and it is close to natural noise. It is the Gaussian noise. The second is an impultionnel noise. It is the noise "Salt and Pepper". The third is the periodic noise. All these noises are noted $N(x, y, n)$ and they are added to the original video $V_{o}(x, y, n)$. The noisy video $V_{n}(x, y, n)$ is described by this equation:

$$
V_{n}(x, y, n)=V_{o}(x, y, n)+N(x, y, n)
$$

\section{A. Application of periodic noise \\ 1) Noises definition}

Periodic noise is caused by electrical interference from an electromechanical device of the sensor. It is an additive noise noted $N(\omega, \theta, \alpha)$ with $\omega$ representing their frequency, $\theta$ is their angle and $\alpha$ represents their amplitude [13]. In our instance, we used a noise defined by a frequency $\omega=0.9$ and an angle $\theta=\{0, \ldots, 640 * \pi\}$ and amplitude $\alpha=500$. We compute the PSNR values between the original and the noisy video. Its variation is described in the following figure:

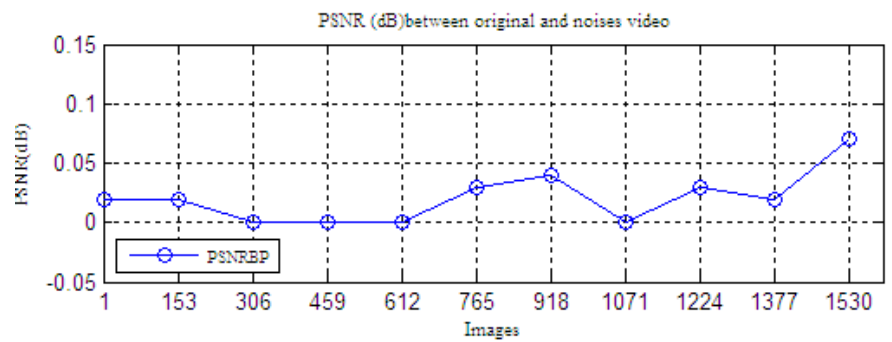

Fig. 1 : PSNR (dB) variation between original and noisy video

\section{2) The uses morphological models}

For this application, we execute a high number of tests to show the performance of our method. We chose to use the morphological models defined in the following table for the periodic noise:

\begin{tabular}{|c|c|c|}
\multicolumn{3}{|c|}{ Table 1: Model used for study the periodic noise } \\
\begin{tabular}{|c|c|c|}
\hline $\begin{array}{l}\text { Model } \\
\text { name }\end{array}$ & PSNR symbol & Models selected \\
\hline 1 & PSNR1 & Close ('square',2)// Open ('square',2) \\
\hline 2 & PSNR2 & $\begin{array}{c}\text { Open (square, } 2) / / \text { Close (disk,1)// Open } \\
\text { (square, 3) }\end{array}$ \\
\hline 3 & PSNR3 & $\begin{array}{c}\text { Close ( square,4) // Open (disk,1)// Close } \\
\text { (square,2) }\end{array}$ \\
\hline 4 & PSNR4 & $\begin{array}{c}\text { Open (square, } 2) / / \text { Close (line, } 4,4) / / \\
\text { Open (square, 2) }\end{array}$ \\
\hline P3 & PSNRP3 & $\begin{array}{c}\text { Open(square,2)//Close(disk,1)// Open } \\
\text { (square,3) }\end{array}$ \\
\hline P4 & PSNRP4 & Open (disk,1)//Close (square,3)// Open \\
$($ line,3,3)
\end{tabular} \\
\hline
\end{tabular}

3) Denoising video by low pass filter followed by morphological filter

First, we applied the 2- FIR low pass filter in special domain to reduce noise in the video image. Secondly, we analyzed the filtered video by morphological operations. The variation of $P S N R$ value in $d B$ defined in the following table and figure:

Table 2: PSNR means (dB) of denoised video

\begin{tabular}{|c|c|c|c|c|c|}
\hline Modeles & PSNR1 & PSNR2 & PSNR3 & PSNR4 & PSNRF \\
\hline PSNR means(dB) & 26.53 & 31.52 & 28.31 & 29.67 & 24.78 \\
\hline
\end{tabular}

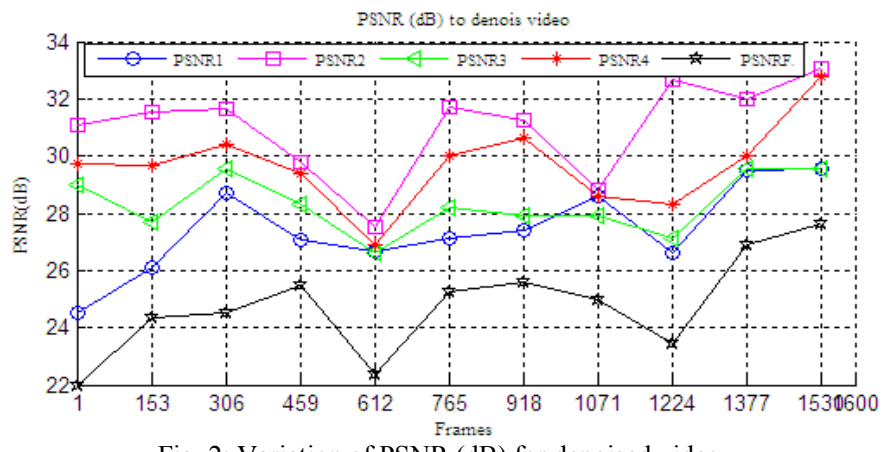

Fig. 2: Variation of PSNR (dB) for denoised video 


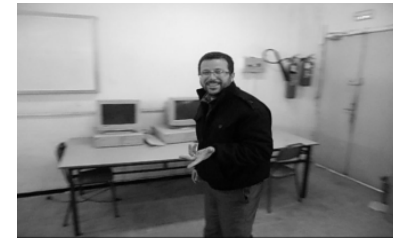

Fig. 3: Original frame

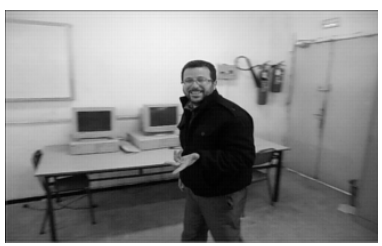

Fig. 5: Filtered frame with low pass filter

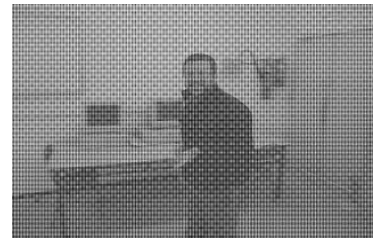

Fig. 4: Noised frame

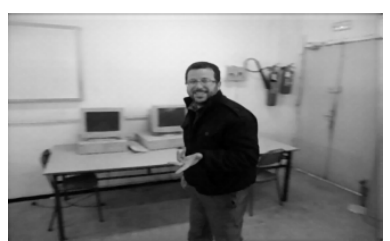

Fig. 6: Filtered frame with middle 2
4) Denoising video by morphological filter followed by low pass filter

In this simulation a waterfall between two different morphological models used in the previous paragraph is highlighted. The outputs of the morphological chain are put to the input of2- FIR filter. An evaluation of PSNR value gives the following results.

Table 3: PSNR (dB) of the combination of morphological operations followed by pass filter

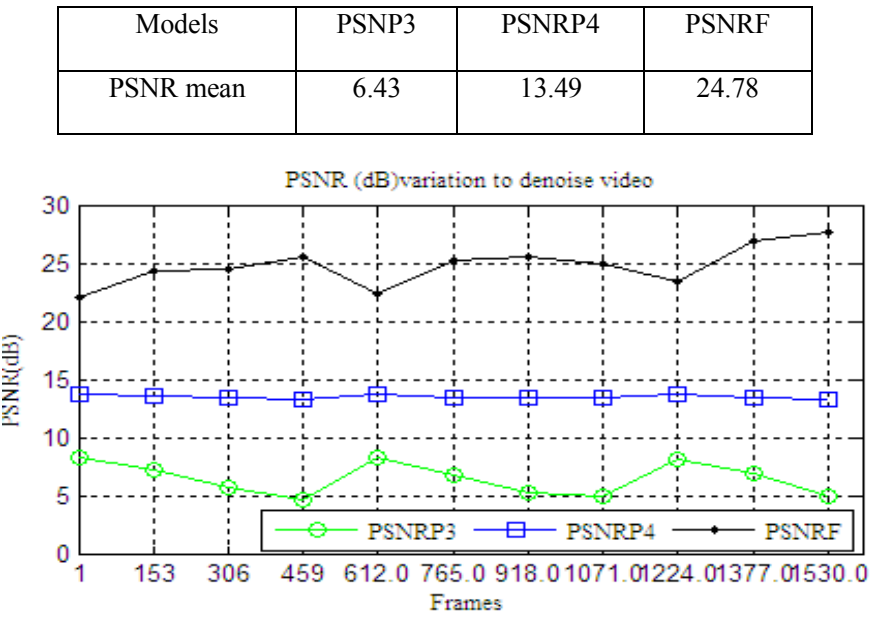

Fig. 7: $P S N R$ of experimental results

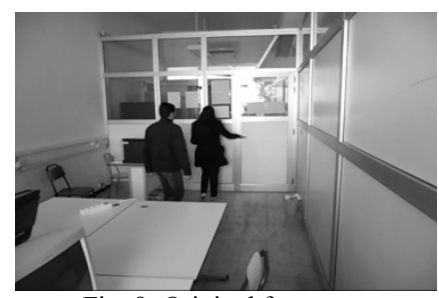

Fig. 8: Original frame

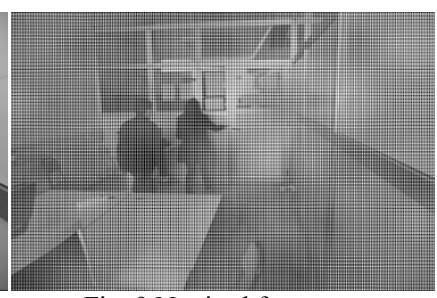

Fig. 9:Nnoised frame

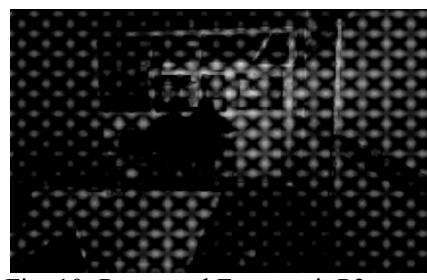

Fig. 10: Processed Frame wit P3 PSNR=6.43dB

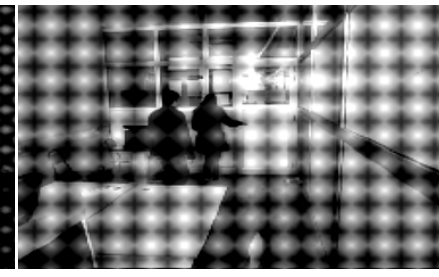

Fig. 11: Processed Frame wit P4 PSNR $=13.49 \mathrm{~dB}$

\section{5) Discussion}

The presented results in Table 4, the figures Fig. 9 and Fig. 13 show the efficiency of the morphological operation in a pretreatment post. It increases the PSNR value between 27.44 $d B$ and $6.837 d B$ of the filtered video. This increase is caused by the performance of mathematical morphology to analyses the contours and geometric propriety of objects. Also, the noise is present by square shapes. That helps the operator morphological to properly analyze. Table 5 and Figure 9 show that the PSNR of the two models that integrate mathematical morphology before the low pass filter in the process of filtering have a very low PSNR compared to that of the video processed by the low-pass filter only. This shows that the implementation of the morphological pretreatment is not effective to denoise video. These results watch the morphological filters cannot filter the periodic noise because it is not an impultionnel noise, and it described by noisy blocks of size upper than the size of structural element. However, increasing the size of the structuring element will destroy the image details.

\section{B. Application of Gaussian noise \\ 1) Noises definition}

It is a noise similar to natural noise. It is described by the random values and variation over time. A noisy video by Gaussian noise is described by this equation:

$V_{N}(x, y, n)=V_{o}(x, y, n)+N(x, y, n)$

$N(x, y, n)$ is defined with a variance $\sigma^{2}$ equal to one and zero mean value $\mu$. In our work, we used an additive Gaussian noise with zero mean and variance equal to $0.03\left(\sigma^{2}=0.03\right)$. The application of this noise gives these results:

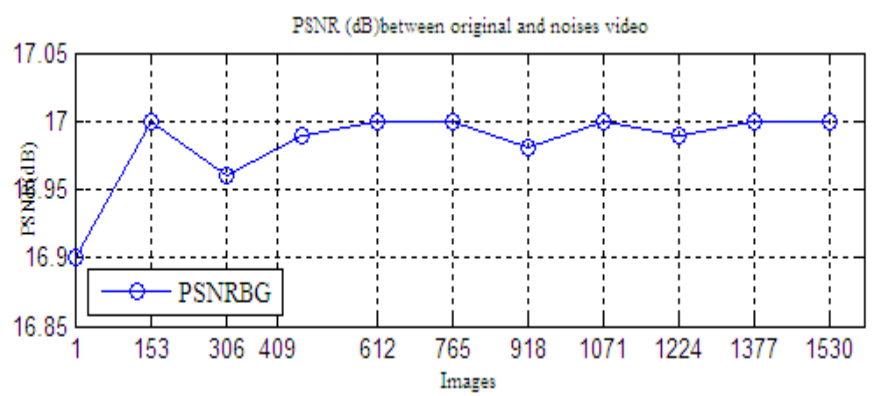

Fig. 12: Variation of PSNR (dB) between the original and the noisy video

\section{2) Used morphological model}

We used these morphological models to reduce Gaussian noise: 


\begin{tabular}{|c|c|c|}
\hline Model names & PSNR symbols & model chose \\
\hline $\mathrm{g} 1$ & PSNRg1 & $\begin{array}{c}\text { Close(square,1)//Open(square,2)// } \\
\text { Close(square,1) }\end{array}$ \\
\hline $\mathrm{g} 2$ & PSNRg2 & $\begin{array}{c}\text { Open(disk,1)//Close(square,1)// } \\
\text { Open(line,2,2) }\end{array}$ \\
\hline $\mathrm{g} 3$ & PSNRg3 & $\begin{array}{c}\text { Close(square,1)//Open(square,2)// } \\
\text { Close(square,1) }\end{array}$ \\
\hline $\mathrm{g} 4$ & PSNRg4 & $\begin{array}{c}\text { Open(disk,1)//Close(square,1)// } \\
\text { Open(line,2,2) }\end{array}$ \\
\hline
\end{tabular}

3) Denoising video by Gaussian low pass filter followed by the morphological operators

A cascade of low-pass filter followed by morphological models to reduce Gaussian noise of variance between $22 \mathrm{~dB}$ and $30 \mathrm{~dB}$ gives the following results:

Tableau 5: PSNR (dB) of the filtering by the Gaussian filter followed by morphological operations

\begin{tabular}{|c|c|c|c|}
\hline models & PSNRg1 & PSNRg2 & PSNRF \\
\hline PSNR means $(\mathrm{dB})$ & 27.37 & 27.17 & 27.04 \\
\hline
\end{tabular}

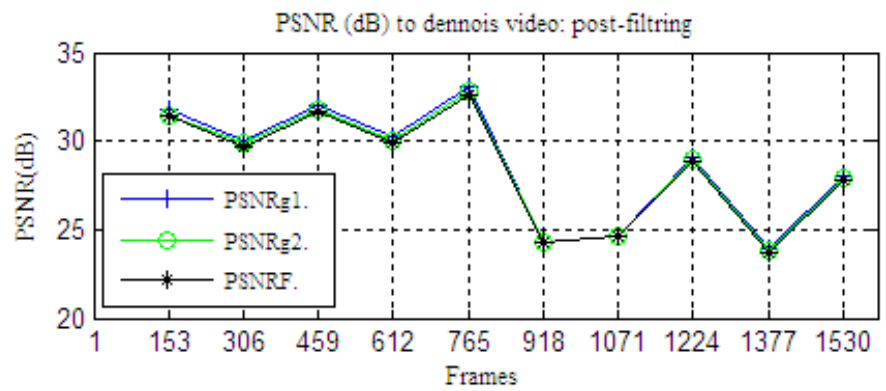

Fig. 13: Presentation of the variation of PSNR (dB)

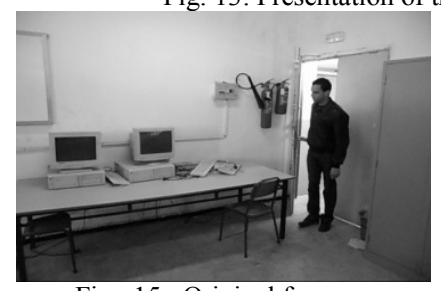

Fig. 15 : Original frame

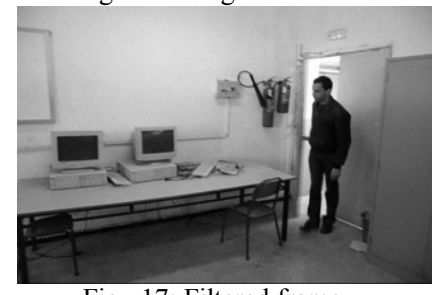

Fig. 17: Filtered frame PSNR $=27.04 \mathrm{~dB}$

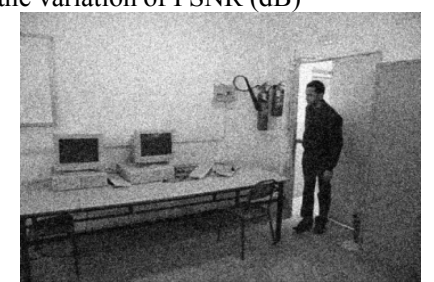

Fig. 16: Noised frame

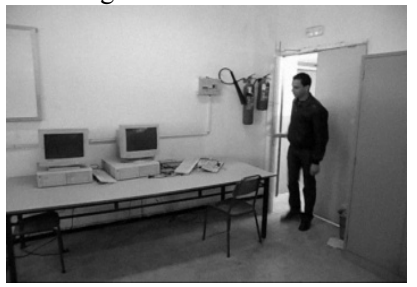

Fig. 18: Processed frame with g1 PSNR $=27.37 \mathrm{~dB}$
4) Denoising video by Gaussian low-pass filter followed by morphological operators

The evaluation of PSNR gives the following result:

Tableau 6: Cascade morphological followed by a Gaussian filter for de-noising video corrupted with Gaussian noise

\begin{tabular}{|c|c|c|c|}
\hline Modeles & PSNRg3 & PSNRg4 & PSNRF \\
\hline PSNR means (dB) & 26.15 & 26.03 & 25.79 \\
\hline
\end{tabular}

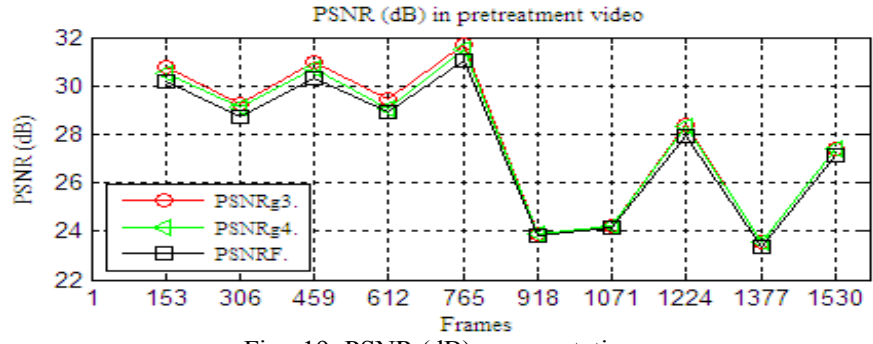

Fig. 19: PSNR $(\mathrm{dB})$ representation

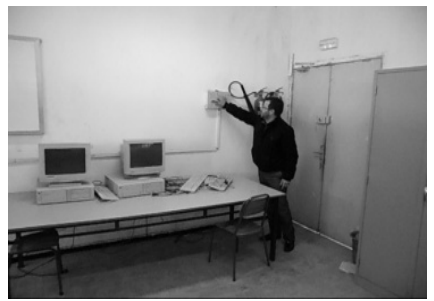

Fig. 20: Original frame

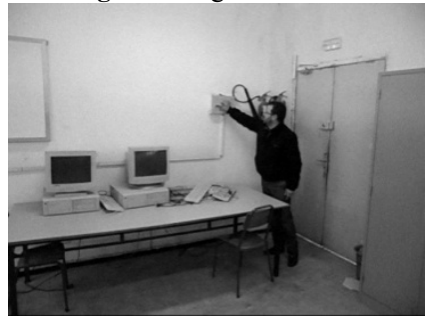

Fig. 22: Processed frame with g3 PSNR $=26.15 \mathrm{~dB}$

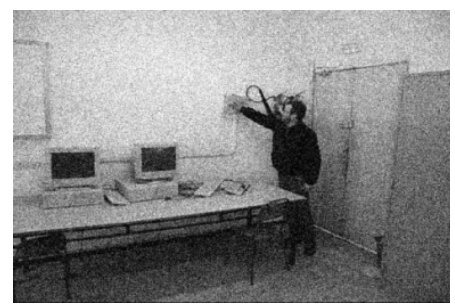

Fig. 21:Noises frame

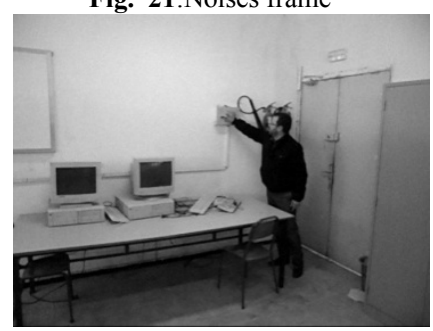

Fig. 23: Processed frame with g4 $\operatorname{PSNR}=26.03 \mathrm{~dB}$

\section{5) Discussion}

Tables 5 and figure Fig. 14 show that the application of the Gaussian filter before morphological operations can increase the PSNR with $0.13 d B$ and $0.33 d B$ and a reverse composition increases the PSNR value with $0.24 d B$ and $0.36 d B$. This performance tells the ability of morphological operations to filter the impultionnel noise and correct the degradation edges of objects. PSNR value varies by small differences between the morphology and the low pass filtering application because the noise does not alter the forms and does not degrade the edges of objects. The performance of mathematical morphology to reduce Gaussian noise is caused by the ability of morphological operation to detect the noisy points. Dilation detects the maximum intensity in the area of the structural element so that the erosion detects the minimum intensity. Therefore the opening and closing allows the removal of the Gaussian noise which explains the validity of using the positions before or after morphological filters.

The opening operator removes Gaussian noise. But, at the same time, when applying erosion, it amplifies the noise. So, it is necessary to apply a closing operation to remove the additional noise on video.

\section{General Discussion}

Morphological filters are used to remove noise without losing image video details. These filters have a directional characteristic which depends on the use of the structural element. The change of the structural element size results is a multi-scale morphological filtering. They allow spatial separation of local variability to differences in function. By opening and closing operators ("Open-Close" successive or 
"Close-Open" successive) both morphological filters compounds remove the noise. However, the size of the noise which is larger than the size of structural element is not removed. For these reasons, the filtered video seems to have some undesirable characteristic present by black blocks when applying "Open-Close" filters or white blocks when applying $\mathrm{f}$ "Closing-Opening" filters. These noise blocks can not be removed by the morphological analyzer because the size of its blocks is larger than the structural element size. Thus, a large size of structural element will destroy the object contours and the geometric characteristics of video images. While a small size of the structural element preserves video image details.

The opening operator application is equivalent to an application of erosion operator. It eliminates the full details of video image not contained in the structural element and the other structures are changed. Next, we applied dilation operator with the same structural element to remove this modification in object. The application of erosion is used to reduce higher frequency noise. It erodes the edges of the moving area. The application of dilation operation on an eroded image extended the movement areas in a video. Opening morphological disappear small details as the image. It maintained the forms presented by fine black lines. It compacts homogeneous white areas. Closing mouth the bottom portions of the image does not contain in the structural element. It removes small lakes. It globes narrow holes. It makes the delicate forms and smaller black areas disappear. It complements the defect forms.

A large size of the structural element eliminates noise but it loses more details of processed objects. On the other side, a small size of the structural element reduces noise and preserves geometrical properties of objects, but, with a slower computation time.

\section{CONCLUSION}

In order to overcome the problems of video filtering and respect the aspects of a multimedia propriety, we have proposed in this paper, a new approach for real time filtering video. We also have combined the standard low pass filter and the mathematical morphology to avoid the noisy real time video without estimating motions. This method may avoid the difficulty faced by the process of motion estimation and avoid successfully all types of noise applied to all video kinds. As we have integrated multimedia format after every treatment, we could, as well, integrate the effect of motion without error.

With application we have found out that there must be a good choice of morphological models, shapes and size of structural elements to get successful filtered real time video.

With its robustness and compatibility with all real time video, this method could be used to define a mathematical relation of motion between frames in real time video.

\section{REFERENCES}

[1] A.Acharya and S.Meher, "Robust Video Denoising for Better Subjective Evaluation », International Conference on Image Information Processing", IEEEJ.C., 978-1-61284-861$7 / 11,2011$

[2] J.C. Brailean, P.Kleihorst, S.Efstratiadis, AK. Katsaggelos and R.L.Lagendijk, "Noise Reduction Filters for Dynamic image
Sequences: A Review », IEEE, VOL. 83, NO. 9, SEFEMBER 1995.

[3] E.J. Balster, Y.F. Zheng, and Robert L. Ewing, "Combined Spatial and Temporal Domain Wavelet Shrinkage Algorithm for Video Denoising", IEEE transactions on circuits and systems for video technology, Vol. 16, No. 2, Feb. 2006.

[4] A. Buades, B. Coll and J.M. Morel, "Denoising image sequences does not require motion estimation", IEEE, 0-78039385-6/05, 2005.

[5] M.Bertalmío, V.Caselles and Á.Pardo, "Movie Denoising by Average of Warped Lines", IEEE transactions on image processing, vol. 16, No. 9, September 2007

[6] M.Ghoniem, Y.Chahir and A.Elmoataz, "Video denoising via discrete regularization on graphs", IEEE Trans, 978-1-42442175-6/08, 2008.

[7] B. Gangamma, S K .Murthy, P.Chandra G C, S. Kaushik and S. Kumar, "A Combined Approach for Degraded Historical Documents Denoising Using Curvelet and Mathematical Morphology, IEEE, 978-1-4244-5967, 2010.

[8] Z.Ji, H.Liao, X.Zhang and Q.H. Wu, "Simple and Efficient Soft Morphological Filter in Periodic Noise Reduction", IEEE, 14244-0549-1/06, 2006.

[9] T.Kombe and R.J.ASSAKO, «morphologie mathématique appliquée aux images rso pour la différentiation des tissus urbains : cas de la ville de douala (cameroun) », vol. 7, $\mathrm{n}^{\circ}$ 1-2-34, p. 251-270, le 23 juillet 2007

[10] P.Kuosmanen, L.Koskinen, and J.Astola," Detail Preserving Morphological Filtering", Tampere University Department of Mathematical Sciences Tampere, Finland, IEEE, 0-8186-29207/92, 1992.

[11] R.Orban Mahmoud, Mohamed T. Faheem and Amany Sarhan, "Intelligent Denoising Technique for Spatial Video Denoising for real-time applications ", IEEE, 978-1-4244-2116-9/08, 2008.

[12] H.Phelippeau, «Méthodes et algorithmes de dématriçage et de filtrage du bruit pour la photographie numérique », thèse doctorat, NXP Semi-conductors Université de Paris Est doctorale ICMS ESIEE, IGM, Lab. Info, A3SI, le 3 avril 2009.

[13] M. I.Sezan, M.K. Ozkan and S.V. Fogel, "Temporally adaptive filtering of noisy image sequences using a robust motion estimation algorithm”, IEEE, CH2977.7/91/0000-2429, 1991.

[14] H. Seddik, and E.B. Braiek "Efficient Noise Removing based Optimized Smart Dynamic Gaussian Filter" International Journal Of Computer and Applications, Vol. 51, N=5 ,pp.1-13, 29 August 2012

[15] Z.Ji, H.Liao, X.Zhang and Q.H. Wu, "Simple and Efficient Soft Morphological Filter in Periodic Noise Reduction", IEEE, 14244-0549-1/06, 2006. 